\title{
Presumed ocular tuberculosis in the United Kingdom: a British Ophthalmological Surveillance Unit (BOSU) study
}

\author{
Katherine Shirley ${ }^{1} \cdot$ Samir Dowlut $^{1} \cdot$ Julie Silvestri $^{1} \cdot$ Carlos Pavesio $^{2} \cdot$ Barny $_{\text {Foot }}{ }^{3}$
}

Received: 3 July 2019 / Revised: 16 October 2019 / Accepted: 4 November 2019 / Published online: 2 January 2020

(c) The Author(s), under exclusive licence to The Royal College of Ophthalmologists 2020

\begin{abstract}
Introduction Ocular tuberculosis (TB) is an extrapulmonary manifestation of mycobacterium infection that most commonly presents as uveitis. This is the first prospective incidence study of presumed ocular tuberculosis performed in the United Kingdom (UK).

Method New cases of ocular tuberculosis presenting to hospitals in the UK were prospectively ascertained between October 2016 and November 2017 with the aid of the British Ophthalmological Surveillance Unit (BOSU). Initial presentation data and 1-year follow-up data was collected using questionnaires.

Results Forty-eight patients were recruited giving an overall incidence for ocular TB of 0.73 per million population per annum. The origin of birth for $71 \%$ of the patients was a non-UK country and $87.5 \%$ had their initial diagnosis of TB made by an ophthalmologist. The most common first line treatment was isoniazid, rifampicin, ethambutol and pyrazinamide which $71 \%$ of patients were treated with $60 \%$ of patients were commenced on a reducing course of oral steroids. At 1-year followup, 29 patients (83\%) had complete resolution of active clinical signs. Mean best corrected visual acuity (BCVA) at presentation was $+0.41 \operatorname{LogMAR}(\mathrm{SD}=0.62)$, compared to $+0.31 \operatorname{LogMAR}(\mathrm{SD}=0.56)$ at 12-month follow-up.

Discussion It is increasingly the responsibility of the ophthalmologist to diagnose ocular TB and although it remains a rare condition, consensus on diagnostic criteria and treatment is required. Increasing recognition and accessibility to gammainterferon testing should enable earlier detection. Treatment with quadruple ATT treatment regimens for at least 6 months shows good clinical outcomes. However, it is still unclear whether steroid use is beneficial. Further large studies with longer follow-up would be warranted to answer these questions.
\end{abstract}

\section{Introduction}

Tuberculosis (TB) is an airborne infectious disease (ID) caused by mycobacterium TB which most commonly affects the lungs [1]. It is estimated that $23 \%$ of the world's population are infected with latent TB [2]. It is a major concern of the World Health Organisation (WHO) as although treatment is successful in $82 \%$ of cases, there is an increasing proportion of drug resistant strains [2].

Of those patients with TB, 16-58\% are found to have extrapulmonary involvement, which includes intraocular

Katherine Shirley

kshirley01@qub.ac.uk

Royal Victoria Hospital, Belfast, UK

Moorfields Hospital, London, UK

Royal College of Ophthalmologists, London, UK presentations [3]. Intraocular TB most commonly presents as uveitis due to the high regional oxygen tension within the ciliary body and choroid although clinical findings can vary significantly. This plays a major role as the primary mechanism for TB to spread to the eye is via haematogenous spread [4]. It can also present due to direct local invasion or as a hypersensitivity response to infection elsewhere. But regardless of the presentation, multiple recurrences in inflammation despite treatment should lead to the consideration of TB as a cause [5].

Obtaining a definitive diagnosis of ocular TB can be difficult. The diagnosis is made more difficult as many patients have no systemic symptoms and it is now believed that a large proportion have latent TB. It is now well recognised that chest X-ray has poor sensitivity for detecting latent TB lesions and high-resolution CT can detect up to $60 \%$ of cavities that were not detected on chest X-ray [6]. Therefore, when investigating for latent $\mathrm{TB}$, high-resolution $\mathrm{CT}$ chest is now recommended. To make the diagnosis of 
ocular TB, obtaining positive polymerase chain reaction assays on ocular fluid would be the most robust method of diagnosis. However, this is not always possible in the clinical setting and current assays have a relatively low sensitivity [7]. Therefore, current practice is to make a presumed diagnosis based on a positive interferon gamma release assay with supporting clinical findings. This is strengthened further by the resolution or improvement of clinical findings following anti-TB treatment.

It is now generally accepted that these patients should receive four-drug anti-TB treatment (isoniazid, rifampicin, ethambutol, and pyrazinamide) for a minimum of 6 months [8]. An area of remaining uncertainty with regard to treatment is the pattern of topical or oral steroid use.

The reported incidence of ocular TB is dependent on the population being studied and ranges from 1.4 to $18 \%$ [4]. Therefore, the objectives of this study were to determine the incidence and distribution of ocular TB in the United Kingdom (UK) as well as typical presenting features. This study will also look at variation in treatment and any relation to clinical and visual outcomes.

\section{Methods}

New cases of presumed ocular TB presenting to hospitals in the UK were prospectively ascertained with the aid of the British Ophthalmological Surveillance Unit (BOSU) [9]. BOSU fund and facilitate research into rare eye conditions that hold significant public health importance such as ocular TB. BOSU uses a monthly reporting card system that is distributed to all consultants and associate specialist Ophthalmologists in the UK.

Respondents indicate how many cases of presumed ocular TB they have seen each month based on the case definition which was included with the card.

The protocol and case definition for presumed ocular TB was reviewed by the BOSU steering committee. The case definition for presumed ocular TB is, "Any patient with evidence of presumed ophthalmic TB who have been commenced on anti-TB treatment". Ophthalmologists were asked to report any cases that were in keeping with this definition between October 2016 and November 2017.

Once a case was reported, the ophthalmologist concerned was sent an initial questionnaire requesting data on demographics, clinical presentation, risk factors, investigations, and initial treatment. For those who responded to the initial questionnaire a subsequent follow-up questionnaire was sent 12 months later, requesting information on clinical outcome, visual outcome and treatment received. Ophthalmologists who did not respond were sent a reminder letter a month after the initial questionnaire was sent.
The data from the questionnaires were collated on a database and were analysed using SPSS statistics software (SPSS, v24; SPSS Inc. Chicago, IL).

Visual acuity (VA) was reported mainly using Snellen VA which was subsequently converted to logarithm of the minimum angle of resolution (LogMAR) [10]. Nonnumerical acuities were converted using a previously described arbitrary scale [11]: counting fingers 1.7, hand movements 2.0, perception of light 2.3 , and no perception of light 3.0.

\section{Results}

During the 12-month collection period 77 BOSU report cards were submitted. Forty-eight of the 77 questionnaires sent were returned (62\% response rate).

\section{Demographics}

This BOSU study recruited 48 patients over 12 months (28 males, 20 females). The mean age was $49(\mathrm{SD}=18)$ (range $12-89$ years old).

Figure 1 outlines the location of reported cases in the UK. The greatest number of patients reported were from the North West of England with all but one of these patients being reported from the city of Manchester. This gives Manchester an incidence of presumed Ocular TB of 2.2 people per 100000 [1]. London was the city with the second highest number of reports with 8 patients. And Belfast and Newcastle had 4 reports each.

Table 1 gives the incidence of presumed ocular TB, based on the population estimates for 2017, for the different countries of the UK. The overall incidence for the UK was 0.73 per million population per annum [1]. The incidence found for England, Scotland and Wales was proportional to their population. The incidence for Northern Ireland was higher than that expected for the proportion of the population.

The origin of birth for $71 \%$ of the patients was a non-UK country, with the majority from Pakistan, India or Africa. Of the patients born in the UK a further $30 \%$ were of nonCaucasian ethnicity (Table 2).

Of the affected patients $27 \%$ had documented Bacillus Calmette-Guérin (BCG) vaccine. None had reported social risk factors such as homelessness, drug use or imprisonment. However, 50\% had known exposure to others with TB infection. This exposure ranged from exposure to family members, friends, orphanage and at work as healthcare professionals. Despite this, only $6(12.5 \%)$ had been previously diagnosed with TB. Therefore $87.5 \%$ had their initial diagnosis of TB made on the basis of their ophthalmic presentation. 


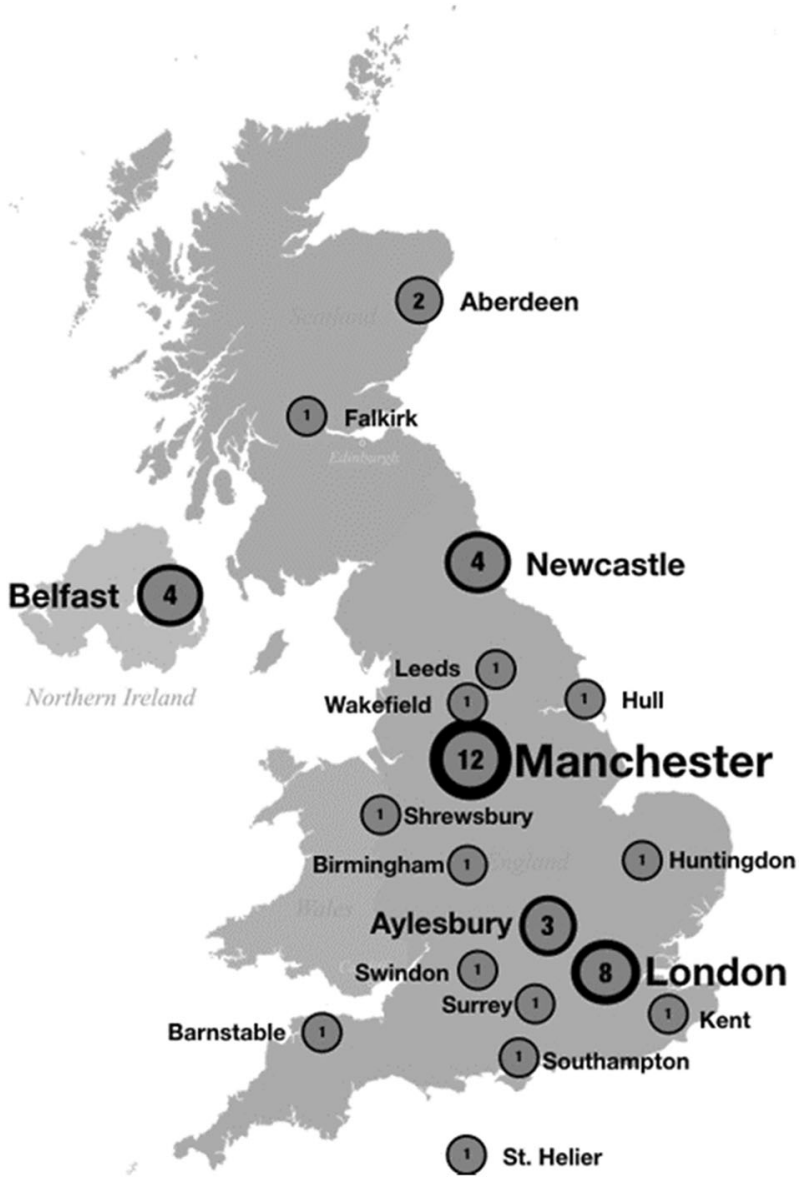

Fig. 1 Location and number of reports.

Table 1 Incidence of presumed ocular tuberculosis in countries in the UK.

\begin{tabular}{ll}
\hline Country & Per million population per annum \\
\hline England & 0.7 \\
Scotland & 0.6 \\
Wales & 0 \\
Northern Ireland & 2.1 \\
\hline
\end{tabular}

Of the 48 patients, 24 patients had bilateral signs, 10 patients had only right eye affected and 10 patients had only left eye affected. This resulted in 68 eyes affected altogether.

\section{Presentation}

In many cases there was a significant delay between onset of symptoms and diagnosis of presumed ocular TB. On average it was 11 months $(\mathrm{SD}=20)$ between symptoms presenting and diagnosis being made, range 1-92 months.

The initial questionnaires revealed that the most common presenting complaint was anterior uveitis in 22
Table 2 Country of birth for patients with presumed ocular tuberculosis in the UK.

\begin{tabular}{ll}
\hline Country of birth & Number of patients \\
\hline UK & 14 \\
Pakistan & 11 \\
India & 7 \\
Africa & 7 \\
Balkans & 2 \\
Asia & 2 \\
Spain/Portugal & 2 \\
Saudi Arabia & 1 \\
Brazil & 1 \\
Unknown & 1 \\
\hline
\end{tabular}

patients (45.8\%), however, only $32 \%$ of these had anterior uveitis alone. Of all patients 19 patients $(40 \%)$ had an intermediate uveitis and 19 patients (40\%) had a panuveitis. Retinitis or vasculitis was reported in $11(23 \%)$ of patients. Only $2(4.1 \%)$ patients had a neuroretinitis or optic neuropathy and $2(4.1 \%)$ patients presented with a scleritis. Three patients were reported as having another clinical signs: one with vitreous haemorrhage, one with choroidal effusions and one with a limbal phlycten (Fig. 2). The average vision at presentation of all eyes was LogMAR $0.39(\mathrm{SD}=0.57)$.

All patients had a positive IGRA result but only 13 patients (27\%) had a Mantoux test performed, one of which was negative. A HIV test was documented in 32 patients $(67 \%)$ and none of these showed a positive result. Nine patients (19\%) had a tissue biopsy performed; seven of these were lymph node biopsies reported as a positive result in $71 \%$ of cases (test unspecified) and two were vitreous biopsies with a $50 \%$ positive result rate.

\section{Treatment}

The most common first line treatment was isoniazid, rifampicin, ethambutol, and pyrazinamide which $71 \%$ of patients were treated with. Sixty percent of patients were commenced on a reducing course of oral steroids. Twentynine percent of these patients had been on oral steroids prior to commencing anti-TB medication. Fifty percent commenced oral steroids at the same time as their anti-TB medication. And $21 \%$ of patients were not commenced on oral steroids until they had been on anti-TB medication for 1-3 months.

The 1-year follow-up questionnaire was received for 35 patients (72.9\% response rate). Two patients had been lost to long-term follow-up. Thirteen patients (37\%) had ongoing treatment at 1-year review. Twenty patients $(57 \%)$ had either completed treatment or been discharged. 
Fig. 2 Presenting complaint.

Presenting complaint

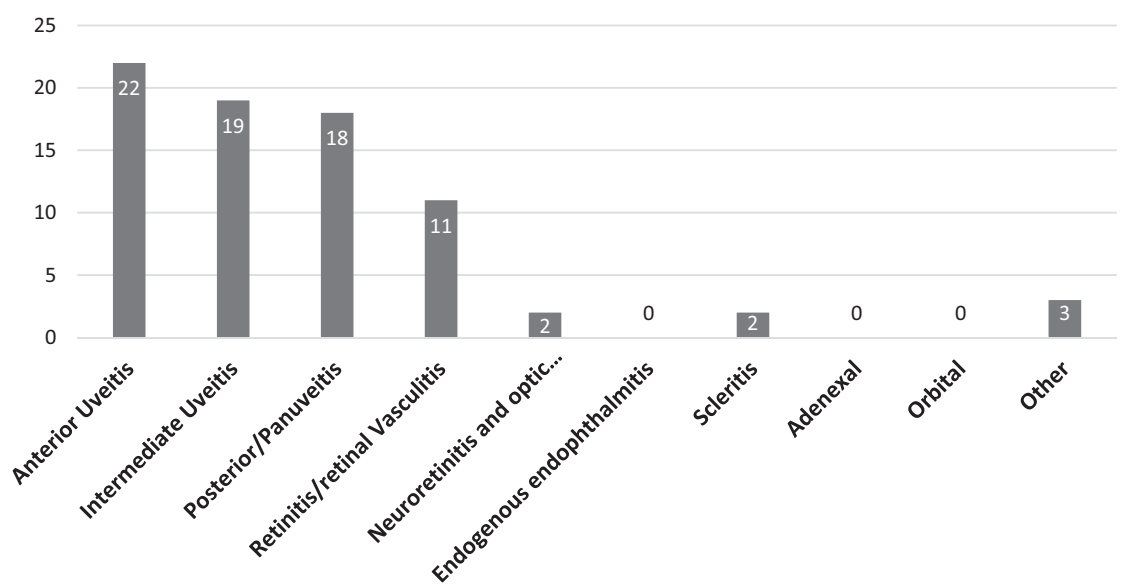

Fig. 3 BCVA change over 12 months.

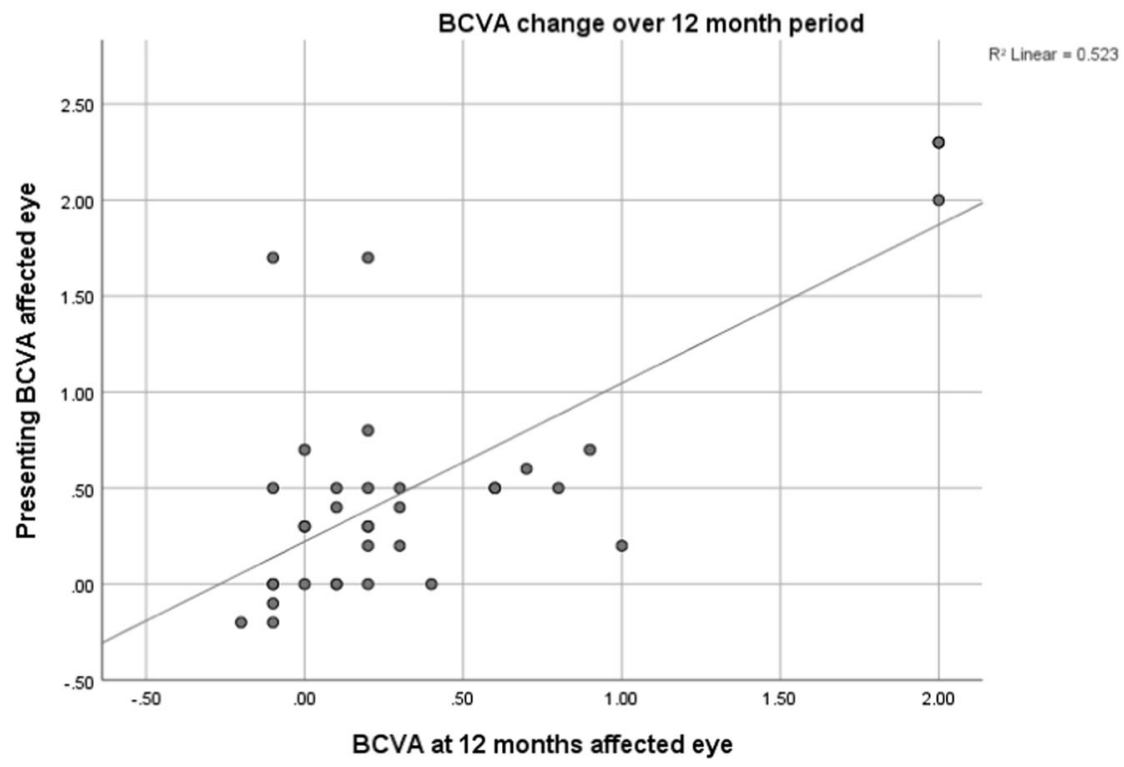

\section{Outcome}

The 1-year follow-up questionnaire revealed that only two patients had no improvement in clinical findings following treatment. Four patients $(11 \%)$ had only partial improvement in clinical signs. Twenty-nine patients $(83 \%)$ had complete resolution of active clinical signs, with 16 patients (46\%) having residual inactive findings.

Twelve patients $(34 \%)$ had no vision loss at 1-year follow-up. Of the 23 patients who had vision loss $65 \%$ were felt to be due to presumed ocular TB with the rest developing ocular sequelae.

For the 50 eyes with 12-month follow-up, the mean BCVA at presentation was $+0.41 \operatorname{LogMAR}(\mathrm{SD}=0.62)$, compared to $+0.31 \operatorname{LogMAR}(\mathrm{SD}=0.56)$ at 12 -month follow-up. This illustrates a mean visual improvement of
0.1 LogMAR unit equating to a gain of one line in Snellen VA (Fig. 3). $48 \%$ of patients had BCVA of 0.1 LogMAR unit or better in the affected eye at 12-month follow-up.

There was a significant correlation between presenting BCVA and change in BCVA over 12 months in affected eyes (Pearson's correlation coefficient $-0.517, p=0.001$ ). There was no statistically significant correlation found between diagnostic delay or steroid use and visual outcome. However, as this was an incidence study the numbers are small and therefore statistical power would not be achieved.

Of particular note are the cases that showed significant visual improvement and those that had poor visual outcome. There were 4 cases that showed significant improvement from worse than 0.5 LogMAR to better than 0.3 LogMAR. Three of these patients had intermediate uveitis and one had a corneal phlycten. All of these patients were diagnosed and 
commenced on treatment within 3 months of symptom onset and all received 6 months of anti- tubercular treatment. Only half were also treated with oral steroids.

There were also 7 patients whose final VA was worse than 0.7 LogMAR. $71 \%$ of these patients presented with choroidal involvement including choroiditis and choroidal effusions. Two patients had a diagnostic delay of over 2 years from symptom onset to diagnosis. Half of the patients were treated with oral steroids and all received at least 6 months of anti-TB treatment. Fifty-seven percent of patients have ongoing activity including cystoid macular oedema or uveitis. 2 Patients have residual macular scarring.

\section{Discussion}

This is the first prospective study of ocular TB in the UK. The annual incidence of presumed ocular TB for the UK based on this study is 0.73 cases per million population [12]. This likely represents the minimum incidence as the BOSU reporting system allows for a certain level of underreporting. Previous BOSU studies have demonstrated ascertainment rates between 75 and 95\% [13]. Although not directly linked to ascertainment the most common way of measuring under ascertainment is to measure response rate [13]. In this study 77 report cards were returned. However, of the 77 questionnaires sent, 48 were returned representing a response rate of $62 \%$.

There have been few incidence studies of ocular TB in non-endemic areas. One recent study reported 8 new cases of ocular TB in 2015 in the Manchester area [14]. By comparison our study reports 12 new cases from Manchester in 2017. If we account for the element of underreporting this would indicate a marked increase in incidence of ocular $\mathrm{TB}$, at least within high incidence areas such as Manchester. It must also be considered that the rise in incidence may partially reflect the growing awareness of TB as a cause for atypical uveitis and choroiditis [15].

It has been reported in some UK cities, Newcastle for example, that the rate of TB as a cause of Uveitis can be as high as $1.5 \%$ [16]. A 10-year retrospective study in Newcastle reported 21 cases of presumed ocular TB, which suggests a yearly incidence of $\sim 2$ patients. This also supports the suggested increased incidence as our study collected 4 new cases from Newcastle in 1 year [16].

Diagnostic confirmation of ocular TB has also greatly improved with the introduction of gamma-interferon testing, which eliminates uncertainty from previous BCG vaccination [17]. The QuantiFERON-TB Gold testing has demonstrated specificity of up to $98 \%$ which is superior to skin testing. It is important to be cautious however as sensitivity is only estimates at $80 \%$, therefore a negative
QuantiFERON-TB Gold test will not rule out active TB disease and clinical suspicion should be considered in these cases [18]. All of the patients in this study had a positive gamma-interferon test.

This study found only $29 \%$ of patients presenting with ocular TB were born in the UK with only 10 patients of white British ethnicity. The majority of patients were born in Pakistan, India, or Africa, representing 11, 7, and 7 cases, respectively. It is well recognised that TB is endemic in Asian countries with India accounting for $20 \%$ of the global TB burden [19]. This finding highlights the importance of establishing country of birth in patients presenting with uveitis as this is a major risk factor for $\mathrm{TB}$ and should prompt further investigation. It is likely also to account for the incidence peaks in Manchester and London were immigration rates are the highest.

Half of the patients in this study had previous exposure to TB but only $13 \%$ had a prior diagnosis. Therefore, $87 \%$ of patients had their diagnosis of TB made based on their ophthalmic presentation. These patients represent a proportion of the $20 \%$ of cases of TB where pulmonary involvement is absent [20]. These patients present a challenge to clinicians as there is lack of agreement between ophthalmologists and ID specialists around diagnostic criteria [21]. The uncertainty between clinicians regarding the role and duration of anti-tubercular treatment (ATT) and corticosteroid use makes management of these patients difficult as respiratory and ID clinicians often look to the ophthalmologist for guidance in those patients who present primarily with ophthalmic findings [7]. The ongoing work by the collaborative ocular tuberculosis study group, is highlighting the differences in treatment strategies and outcomes. Their work will be pivotal in developing best practice guidelines for patients with tubercular uveitis [22]. A cohesive approach to treating these patients will hopefully lead to improved clinical outcome.

A concerning finding of this study is the delay in diagnosis of ocular TB. On average there were 11 months between onset of symptoms and diagnosis of TB. The diagnosis of TB by ophthalmologists can be challenging, however the wider availability of gamma-interferon testing in recent years should lead to improvements. Historically in the UK there has been a lack of awareness of TB as a cause of uveitis as it is a non-endemic region and a rare cause. Increasing rates of immigration and ongoing work in this area are starting to improve awareness and we hope this will reduce diagnostic delay. Interestingly statistical analysis did not demonstrate a correlation between diagnostic delay and adverse clinical outcome in this study.

In this cohort of patients, $90 \%$ of cases presented with an element of uveitis. $32 \%$ had anterior uveitis alone but $40 \%$ had panuveitis and $23 \%$ had a retinitis or vasculitis. It is well recognised that the uveal tract is involved in the 
majority of ocular TB cases as it has the greatest blood flow resulting in haematogenous spread [23]. Our findings concur with the previous studies that have demonstrated that posterior uveitis is the most common presentation [24]. Although uveitis is the most common presentation of intraocular TB, a high index of suspicion should be employed as TB can affect many different intraocular tissues. In our study, there were 2 reported cases of optic nerve involvement and two cases of scleritis, giving an incidence of $4 \%$ for both these presentations. Rare presentations including vitreous haemorrhage, choroidal effusions and conjunctival phlycten were also reported. Any form of ocular inflammation particularly if it is resistant to treatment should increase the level of suspicion for intraocular TB [4].

Our study revealed a variety of ATT regimes and durations. The majority $(71 \%)$ of clinicians used quadruple therapy including isoniazid, rifampicin, ethambutol and pyrazinamide. Sixty-eight percent used ATT for 6 months but it ranged from 2 to 13-month courses. Many could not tolerate 1 or more drugs and treatment was adjusted to allow for this. Systemic treatment is always warranted as although ocular manifestation may be the presenting complaint, pulmonary or other foci of infection may co-exist and, in some cases, the ophthalmic signs are thought to be an immunological response to a distant focus. Further studies regarding ocular penetration are required but oral Rifampicin has been found to reach aqueous levels of up to $9 \%$ which is felt to be therapeutic [23]. Established ATT treatment regimens have been found to be very effective, with previous studies reporting resolution of uveitis in approximately 60-70\% [7]. In our study, $83 \%$ showed resolution of active uveitis. However, it is important to be aware of the rising incidence of drug resistant TB and when treatment response is suboptimal it is advisable to consult ID clinicians to consider alternative treatments [25]. It is important also to be aware of other risk factors for treatment failure such as immunosuppression. Our study only had two patients reported as immunosuppressed which was too small a number to analyse however a retrospective study performed in London demonstrated that patients on immunosuppressive therapy had higher odds of treatment failure [26].

An area of greater variation in treatment is the concomitant use of corticosteroids. Sixty percent of our patient cohort were prescribed corticosteroids at some stage of their treatment. Seventy nine of these patients were already using steroids or were commenced on them at the same time as commencing ATT. Twenty one had oral steroid commencement delayed until ATT was established. There was no statistical difference between clinical outcome dependant on steroid use or timing. Several studies have demonstrated benefit from corticosteroid use with ATT, however some authors suggest delaying steroid treatment to assess response to ATT may be desirable. This has to be considered in relation to the risk of sight loss due to uncontrolled inflammation [24]. The lack of agreed treatment guidelines for ocular TB in the UK and worldwide is a major challenge for clinicians.

In this study, 29 patients (83\%) had complete resolution of active clinical signs at 1 year, $4(11 \%)$ had partial resolution and only two patients $(6 \%)$ had no improvement. There was on average an improvement in VA of $0.1 \mathrm{Log}$ MAR unit, from $+0.41 \operatorname{LogMAR}(\mathrm{SD}=0.62)$ at presentation to $+0.31 \operatorname{LogMAR}(\mathrm{SD}=0.56)$ at 12 -month follow-up. This level of visual improvement is in keeping with similar retrospective studies [14]. There is understandably a positive correlation between presenting BCVA and VA at outcome. This highlights the importance of early recognition and treatment to prevent the sequelae of intraocular inflammation such as cataract and macular oedema which are responsible for the majority of cases of significant visual loss.

The strengths of this study are the prospective nature of case collection and completion of follow-up questionnaires, allowing for false positives to be identified. It is important to take into account the potential for underreporting, bias or loss of data that results from the nature of the BOSU reporting system. Given the rarity of ocular TB there is inevitable low numbers of patients resulting in difficulty in establishing statistically significant correlations between variables.

In summary, ocular TB is a rare ocular presentation of a significant systemic infection. The UK incidence has been estimated at 0.73 per million per annum. Increasing recognition of high-risk groups such immigrants from endemic regions and accessibility to gamma-interferon testing should enable earlier detection of this condition. Treatment with quadruple ATT treatment regimens for at least 6 months shows good clinical outcomes. It is still unclear whether steroid use in these patients is beneficial. Further large studies with longer follow-up would be warranted to answer these questions.

\section{Summary}

\section{What was known before}

- Ocular tuberculosis is a rare cause of uveitis.

- There is no consensus on how best to treat this condition.

\section{What this study adds}

- Incidence of ocular tuberculosis for the United Kingdom is 0.73 per million population per annum. 
- A risk factor for ocular TB is immigrant status with the origin of birth for $71 \%$ of the patients being a non-UK country.

- Eighty-three of patients had complete resolution of symptoms at 12 months follow-up.

\section{Compliance with ethical standards}

Conflict of interest The authors declare that they have no conflict of interest.

Ethical approval Ethics approval was granted by the Office for research ethics committees Northern Ireland (ORECNI) and no patient identifiable information was collected, ref 16/NI/0165.

Publisher's note Springer Nature remains neutral with regard to jurisdictional claims in published maps and institutional affiliations.

\section{References}

1. Jones RM, Brosseau LM. Aerosol transmission of infectious disease. J Occup Environ Med. 2015;57:501-8.

2. World Health Organisation (WHO). Global tuberculosis report. Geneva: WHO; 2018.

3. Davidson JA, Lalor MK, Mohiyuddin T, Loutet MG, Uddin T, Venugopalan S, et al. Tuberculosis in England 2016 Report. London: Public Health England; 2016.

4. Dalvin L, Smith W. Intraocular manifestations of mycobacterium tuberculosis: a review of the literature. J Clin Tuberculosis Other Mycobact Dis. 2017;7:13-21.

5. Sharma A, Thapa B, Lavaju P. Ocular tuberculosis: and update. Nepal J Ophthalmol. 2011;3:52-67.

6. Alkabab Y, Enani M, Indarkiri N, Heysell S. Performance of computed tomography versus chest radiolography in patients with pulmonary tuberculosis with and without diabetes at a tertiary hospital in Riyadh, Saudi Arabia. Infect Drug Resist 2018;11:37-43.

7. Lee C, Agrawal R, Pavesio C. Ocular tuberculosis-a clinical conundrum. Ocul Immunol Inflamm. 2016;24:237-42.

8. Figueira L, Fonseca S, Ladeira I, Duarte R. Ocular tuberculosis: position paper on diagnosis and treatment management. Rev Port Pneumol 2017;23:31-38.

9. Foot B, Stanford M, Rahi J, Thompson J. The British Ophthalmological Surveillance Unit: an evaluation of the first 3 years. Eye. 2003;17:9-15.
10. Beck RW, Moke P, Turpin A, Ferris F. A computerized method of visual acuity testing: adaptation of the early treatment of diabetic retinopathy study testing protocol. Am J Ophthalmol. 2003;135: 194-205.

11. Cochrane T, Silvestri G, McDowell C, Foot B, McAvoy C. Acute retinal necrosis in the United Kingdom: results of a prospective surveillance study. Eye. 2012;26:370-378.

12. Park N. Population estimates for UK, England and Wales, Scotland and Northern Ireland. Office for National Statistics. 2018. www.ons.gov.uk.

13. Imrie FR, Cox A, Foot B, MacEwen CJ. Surveillance of intraocular foreign bodies in the UK. Eye. 2008;22:1141-1147.

14. Krassas N, Wells J, Bell C, Woodhead M, Jones N. Presumed tuberculosis-associated uveitis: rising incidence and widening criteria for diagnosis in a non-endemic area. Eye. 2018;32:87-92.

15. Gan W-L, Jones NP. Serpiginous-like choroiditis as a marker for tuberculosis in a non-endemic area. Br J Ophthalmol. 2013; 97:644-647.

16. Manousaridis $\mathrm{K}$, et al. Clinical presentation, treatment, and outcomes in presumed intraocular tuberculosis: experience from Newcastle upon Tyne, UK. Eye.2013;27:480-486.

17. Itty $S$, et al. Initial results of QuantiFERON-TB Gold testing in patients with uveitis. Eye. 2009;23:904-909.

18. Mori T, Sakatani M, Yamagishi F, Takashima T, Kawabe Y, Nagao K, et al. Specific detection of tuberculosis infection: an interferon-gamma-based assay using new antigens. Am J Respir Crit Care Med. 2004;170:59-64.

19. Agarwal A, Aggarwal K, Gupta V. Infectious uveitis: an Asian perspective. Eye. 2019;33:50-65.

20. Demirci H, Shields CL, Shields JA, Eagle RC Jr. Ocular tuberculosis masquerading as ocular tumors. Surv Ophthalmol. 2004;49:78-89.

21. Conant $\mathrm{M}$, et al. Role of the infectious disease consultant in management of patients with tuberculosis-associated ocular inflammation. Open Forum Infect Dis. 2015;3:1-4.

22. Agrawal R, Gunasekeran DV, Raje, D, Agarwal A, Nguyen, QD, Kon OM, et al. for the Collaborative Ocular Tuberculosis Study Group. Global variations and challenges with tubercular uveitis in the collaborative ocular tuberculosis study. Investig Ophthalmol Vis Sci. 2018;59:4162-71.

23. Albert D, Raven M. Ocular tuberculosis. Microbiol Spectr. 2016;4:1-36.

24. Shakarchi F. Ocular tuberculosis: current perspectives. Clin Ophthalmol. 2015;9:2223-2227.

25. Naidoo A, Niadoo K, McIlleron H, Essack H, Padayatchi N. A review of moxifloxacin for the treatment of drug-susceptible tuberculosis. J Clin Pharmacol. 2017;57:1369-86.

26. Agrawal R, et al. The role of anti-tubercular therapy in patients with presumed ocular tuberculosis. Ocul Immunol Inflamm. 2015; 23:40-6. 